\title{
Guest editorials: Special issue on fog/edge networking for multimedia applications
}

\author{
Yong Jin ${ }^{1} \cdot$ Hang Shen ${ }^{2} \cdot$ Daniele D'Agostino ${ }^{3} \cdot$ Nadjib Achir $^{4} \cdot$ James Nightingale ${ }^{5}$ \\ Published online: 9 September 2019 \\ (C) Springer Science+Business Media, LLC, part of Springer Nature 2019
}

Technological advancements have enabled emerging multimedia applications, which require massive devices with real-time communication, computation, and control. However, many end devices are characterized by limited resources, which identify significant challenges for these devices to provide multimedia services effectively. The traditional cloud Networking paradigm as the de facto centralized big data processing platform is not efficient enough to support many emerging multimedia applications with QoS/QoE/QoI constraints.

Nowadays, fog/edge Networking has become a promising paradigm for multimedia systems. The basic idea is to leverage a multitude of collaborative end devices and near-user infrastructures to carry out a substantial amount of computation, storage, and communication tasks, for enabling low latency, energy efficiency as well as agile computation augmenting services for multimedia. Despite the many benefits and opportunities fog/ edge Networking offers, there are several research and technical challenges that need attention from the research community. Some of these challenges include: how to design an efficient fog/edge Networking based multimedia Networking framework, how to make multimedia systems more intelligent, how to achieve the efficient multimedia data processing and distribution with edge/fog Networking, and how to design an efficient incentive mechanism for performance enhancement.

The aim of this special issue is to focuse on recent advances in architecture, algorithms, optimization, and models for multimedia fog/edge Networking systems. Original, unpublished contributions and invited articles, reflecting various aspects of multimedia systems with fog/edge Networking are encouraged.

The special issue has gone through an open call for papers and rigorous peer-review, where 13 articles from 32 submissions have been selected as representatives of ongoing research and development activities.

The first paper by Yan Chen et al. on "Fog computing support scheme based on fusion of location service and privacy preservation for QoS enhancement" introduced the fog computing into distributed multimedia systems and largescale concurrent multimedia services, and considers the privacy protection of location services, to further solve the problems such as large-scale concurrent multimedia services, etc.

The second paper by Feng Wang et al. on "Heterogeneous mobile network access control technology based on mutual trust mechanism" proposed a heterogeneous mobile network access control technology based on mutual trust mechanism.

This article is part of the Topical Collection: Special Issue on Fog/Edge Networking for Multimedia Applications

Guest Editors: Yong Jin, Hang Shen, Daniele D’Agostino, Nadjib Achir, and James Nightingale

Yong Jin

jinyong@cslg.edu.cn

Hang Shen

hshen@njtech.edu.cn

Daniele D'Agostino

dagostino@ge.imati.cnr.it

Nadjib Achir

nadjib.achir@univ-paris13.fr

James Nightingale

james.nightingale@uws.ac.uk
Changshu Institute of Technology, Changshu 215500, China

2 Nanjing University of Technology, Nanjing 211816, China

3 Institute for Applied Mathematics and Information Technologies, National Research Council of Italy, Via Marini 6, I-16149 Genoa, Italy

4 University of Paris 13, 93430 Villetaneuse, France

5 University of the West of Scotland, Glasgow, UK 
The third paper by Lu Liu on "Multimedia intelligent fog computing scheme based on robust perception for complex networks" combined synchronization control, robustness perception and fog computing in complex networks to improve the quality of multimedia services, and designs a series of architecture and control algorithms for complex networks.

The fourth paper by Yuan Shu et al. on "Green communication mobile convergence mechanism for computing selfoffloading in 5G networks" designed a 5G network architecture for supporting autonomy and deployed the multiple autonomous base stations to solve the problem of large-scale computing, limited resource and low utilization of $5 \mathrm{G}$ network.

The fifth paper by Di Fan et al. on "Time delay estimation algorithm based on virtual Array and MUSIC for single sensor system" presented a novel method of constructing virtual sensor array, and proposed furthermore the MUSIC delay estimation algorithm based on the virtual array.

The sixth paper by Zengzhen Shao et al. on "Identifying influential nodes in complex networks based on Neighbours and edges" proposed the NL centrality based on the neighbors and importance of edges, which considers the second-degree neighbor's impact on the influence of a node and utilizes the connectivity and unsubstitutability of edge to distinguish topological position of a node.

The seventh paper by Yonggang $X u$ et al. on "Queue models for wireless sensor networks based on random early detection" studied the performance analysis models of the WSN node, constructs a WSN node queuing model based on $\mathrm{M} / \mathrm{M} / 1$, a WSN node queuing model with priority, a WSN node $\mathrm{M} / \mathrm{M} / 1$ queuing model based on random early detection (RED) and a WSN node M/M/1 queuing model with priority based on RED.

The eighth paper by Lang $H u$ et al. on "UAVs joint vehicles as data mules for fast codes dissemination for edge networking in Smart City" proposed an Unmanned Aerial Vehicles (UAVs) joint Vehicles as Data Mules for Fast Codes Dissemination (UVDCD) scheme to spread codes as a fast and low-cost pattern for edge networking in smart city.

The ninth paper by Wenjuan Zhang on "A data fusion privacy protection strategy with low energy consumption based on time slot allocation and relay in WBAN" considered the characteristics and node data rate in WBAN and meet the requirements of the reliability of data transmission in the network, to bring the additional energy consumption, and strategy information dynamic relay selection depend on the channel information and energy.

The tenth paper by Zijia Yu on "A multipath routing protocol using congestion control in wireless multimedia sensor networks" designed an improved real-time routing protocol (SPEED-RR) with QoS guarantee based on the SPEED protocol, which is mainly used to make an effective compromise between real-time and energy cost so as to ensuring the real-time performance of QoS.

The eleventh paper by Rongyu Tian et al. on "Complex application identification and private network mining algorithm based on traffic-aware model in large-scale networks" defined the complex applications and distinguished it from traditional protocols, then proposed a traffic-aware model describing the communication process of complex applications.

The twelfth paper by Chunguang Zhang on "Hierarchical resource scheduling method using improved cuckoo search algorithm for Internet of Things" studied and explored the hierarchical resource scheduling of the Internet of Things based on improved heuristic algorithm, then proposed a cuckoo search algorithm based on adaptive Cauchy mutation.

The thirteenth paper by Suqin Wu on "Communication Modulation Recognition Algorithm based on STFT Mechanism in Combination with Unsupervised Featurelearning Network" proposed a novel recognition algorithm based on deep learning network far communication signal features for dealing with the limitations of traditional communication modulation recognition algorithms.

To conclude this special issue, we would like to thank all reviewers who have helped in the paper review process, and the authors for their contribution and efforts to complete the articles with high quality. Finally, we express our gratitude to Prof. Xuemin Shen for initiating this special issue and inviting us to undertake this rewarding activity, as well as Ms. Melissa Fearon for the help during the publication process.

Publisher's note Springer Nature remains neutral with regard to jurisdictional claims in published maps and institutional affiliations.

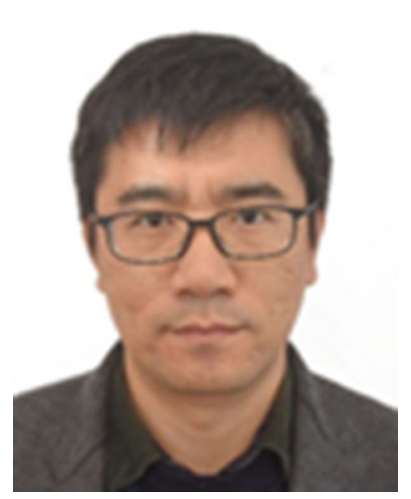

Yong Jin is currently an associate professor in the School of Computer Science and Engineering at Changshu Institute of Technology. He is a China CCF member. His research papers have been published in many conferences and journals such as Journal of Sensors, Journal of Medical Systems, Personal and Ubiquitous Computing, Multimedia Tools and Applications, etc. His recent research has been focused on wireless sensor networks, fog computing, wireless charging system, Industrial Internet of Things, deep transfer learning, etc . 


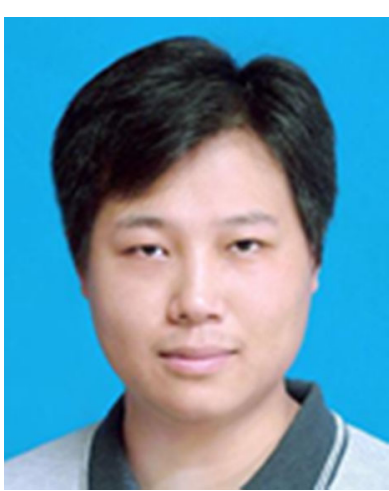

Hang Shen is currently a full-time postdoctoral fellow at the Department of Electrical \& Computer Engineering, University of Waterloo, Waterloo, ON, Canada. He received the Ph.D. degree in Computer Science from the Nanjing University of Science \& Technology. In 2015, he joined Nanjing Tech University, Nanjing, China, appointed as an Assistant Professor in the Department of Computer Science \& Technology. Since 2019, he has been appointed as an Associate Professor. His research interests involve $5 \mathrm{G}$ network slicing and security, and drone communication and networking. He is supported by the National Natural Science Foundation of China, the Natural Science Foundation of Jiangsu Province and other research projects.

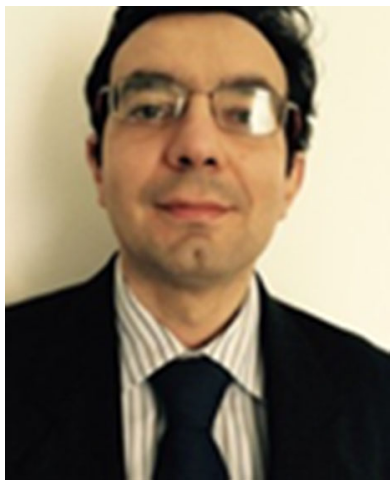

Daniele D'Agostino Ph.D., is a researcher at the Institute of Applied Mathematics and Information Technologies of the National Research Council of Italy. Hisresearch activities concern the design of science gateways in different research fields, the resource allocation in Grid/Cloud environments and the development of parallel software in many research fields. He co-organized the 22th Euromicro International Conference on Parallel, Distributed, and Network-Based Processing (PDP 2014), several special issues on ISI journals and coauthored more than 90 scientific papers, published in journals, book chapters and conference proceedings.

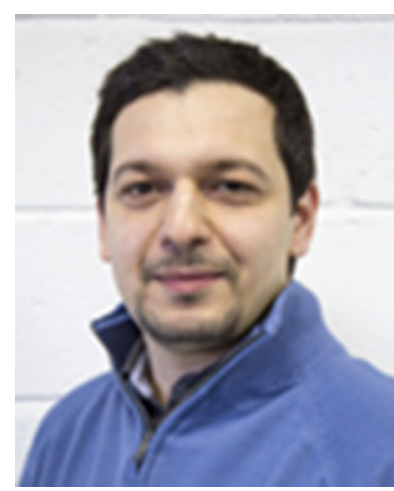

Nadjib Achir is associate professor at University Paris 13 , since 2004. He received his $\mathrm{Ph} . \mathrm{D}$. Computer Science from University of Pierre et Marie Curie (Paris 6) in 2003. In 2011, he obtained INRIA fellowship and worked two years as an associated researcher at Hipercom team. In 2014, he obtained his Habilitation a Diriger des Recherches (HDR) degrees in Computer Science from the University of Paris 13. His research interests cover design, modeling, and optimization of networks and services. Currently, his research focuses cloud, mobile edge and fog computing and autonomous vehicular communications. He is actively involved in the community as a conference chair, TPC member, and a reviewer for many conferences and journals.

James Nightingale is a Postdoctoral Research Associate with the AudioVisual Communications \& Networks (AVCN) Research Centre, working on the EPSRC funded project Enabler for next-generation mobile video applications (EP/J014729/1). His research interests include mobile networks, multihoming and video streaming techniques. He is a member of the IET and IEEE. He received the BSc degree in Network Networking from Edinburgh Napier University, UK with distinction and as winner of the Napier Medal for Outstanding Achievement, and the BSc (Honours) degree in Computer Networks from the University of the West of Scotland (UWS), UK with First Class Honours and won the Best Honours Dissertation Prize. He received his $\mathrm{PhD}$ from UWS, with Outstanding Progression Award. https://www.scopus.com/authid/detail. uri?authorId $=36775133600$ 